\title{
Effect of Needle Characteristic on Fibrous PEO Produced by Electrospinning
}

\author{
Haijun $\mathrm{He}^{1}$, Yahya $\mathrm{Kara}^{1}$ and Kolos Molnar ${ }^{1,2^{*}}$ \\ 'Department of Polymer Engineering, Faculty of Mechanical Engineering, Budapest University of Technology and Economics, \\ Müegyetem rkp. 3, H-1111 Budapest, Hungary \\ ${ }^{2}$ MTA-BME Research Group for Composite Science and Technology, Müegyetem rkp. 3, H-1111 Budapest, Hungary
}

Received: 30 June 2018; accepted: 04 September 2018

\begin{abstract}
The technique of electrospinning has been researched for several decades. Almost all parameters have been investigated in the past years, e.g., solution parameters, process parameters, and environmental conditions. Among the solution parameters, the viscosity of the polymer solution is an extremely important factor for fiber formation and morphology. In general, however, viscosity of the polymer solution is mostly controlled by the solution concentration or by the molecular weight of the polymer in electrospinning field. Herein, we described the reason of a wellknown but not completely explained conclusion that the needle diameter can have an influence on the fiber morphology. In this study, polyethylene-oxide (PEO) with a molecular weight of 400,000 g/mol was dissolved in a mixture of ethanol and water with a proportion of 1:3. The relationship between the viscosity of the polymer solution and shear rate was characterized by a plate-plate rheometer. A shear flow model was discussed, while polymer material was flowing through a needle, which presented that different deformation rates were imposed on materials due to variable needle diameters. Combining the rheological experiments and analysis of the shear flow model, the viscosity of polymer solution flowing out the needle was predicted by needle diameter. Through observing the obtained fibers' morphology by scanning electron microscopy and measuring their diameters by image processing software, it was found that the fiber diameter increased with the increase of needle diameter, as expected, which agreed with the relationship of fiber diameter and polymer viscosity.
\end{abstract}

Keywords: electrospinning, needle diameter, solution rheology, viscosity

\section{Introduction}

From the 1990s, electrospinning was being supposed to be a possible technique to produce fibers with nanometer size diameter, for specific applications, namely, wound dressings, tissue engineering scaffolds, protective clothing, electronics [1], sensors [2], drug delivery materials $[3,4]$, composites $[5,6]$, filters[7], etc.

Needle electrospinning is the oldest and the simplest electrospinning device, which is still commonly used in academia [8]. It mainly consists of a needle and a grounded collector. A high voltage is supplied between the needle and the collector. In the electrospinning process, the electrostatic forces applied on a charged solution overcome the surface tension, and a jet is formed and ejects from the needle tip towards a grounded collector [9]. Electrospinning is a complex method controlled by lots of processing parameters and solution properties in fiber formation. Among these parameters, the viscosity of the polymer solution plays a significant role for fiber morphology and diameter in the electrospinning process. Generally, it is a common way to control the viscosity of the solution by adjusting various properties, such as solution concentration, polymer molecular weight, additives, type of solvent, etc [10].

The experimental data showed a strong dependence on viscosity for fiber morphology [11-13]. It has been found that with very low viscosity, almost continuous fibers are not formed, while with very high viscosity, it is difficult to form polymer jets from polymer solution [14]. The viscosity, solution concentration, and molecular mass of the polymer are correlated to each other [15]. In the case of solution electrospinning, the viscosity of the polymer solution is mainly controlled by the solution concentration and molecular weight. Therefore, the fiber diameter mainly varies with the solution concentration and molecular weight $[8,12,16-18]$.

\footnotetext{
*Author for correspondence: molnar@pt.bme.hu.
}

Although the effect of needle diameter on the fiber morphology has been reported, it was just an empirical result without any further analysis [10]. What is more, many studies have been reported in the literature on the effect of solution viscosity on fiber morphology by controlling properties of the solution; however, the effect of needle size or geometry on the viscosity of polymer solution has not been investigated $[8,11-18]$. When a fluid is forced through a needle, the particles which compose the fluid generally move faster near the needle's axis and slower near its wall; therefore, shear stresses are generated, which can have an influence on the solution viscosity, assuming the liquid as non-Newtonean. Therefore, for needle electrospinning, the needle size, flow rate, and flow speed are the main parameters affecting the solution viscosity. To figure out a rational explanation for the effect of needle size on the fiber morphology from rheological aspect, we obtained the correlation between shear rate and viscosity based on viscosity measurement. Then, we computed the shear rate introduced on polymer solution by different needle diameters at a constant flow speed and flow rate, respectively.

In this study, we investigated the effect of needle diameter, flow speed, and flow (volume) rate on the fiber morphology in the case of nanofibers obtained from poly(ethylene oxide) (PEO) solutions. In theory, the polymer viscosity can be controlled by the needle geometry and the shear conditions.

\section{Materials and Methods}

Materials. Poly(ethylene oxide) (PEO) is often used as a model for the electrospinning process, because it is a watersoluble, easy-to-electrospin and non-toxic polymer $[19,20]$. In this study, PEO with a molecular weight of $400,000 \mathrm{~g} / \mathrm{mol}$ was dissolved in a mixture of ethanol-water (1:3) with the addition of $0.01 \% \mathrm{NaCl}$. PEO solutions with concentrations of $3.5 \%, 4.0 \%$, and $4.5 \%$ were stirred overnight at room temperature using a magnetic stirrer in order to obtain homogeneous solutions. 


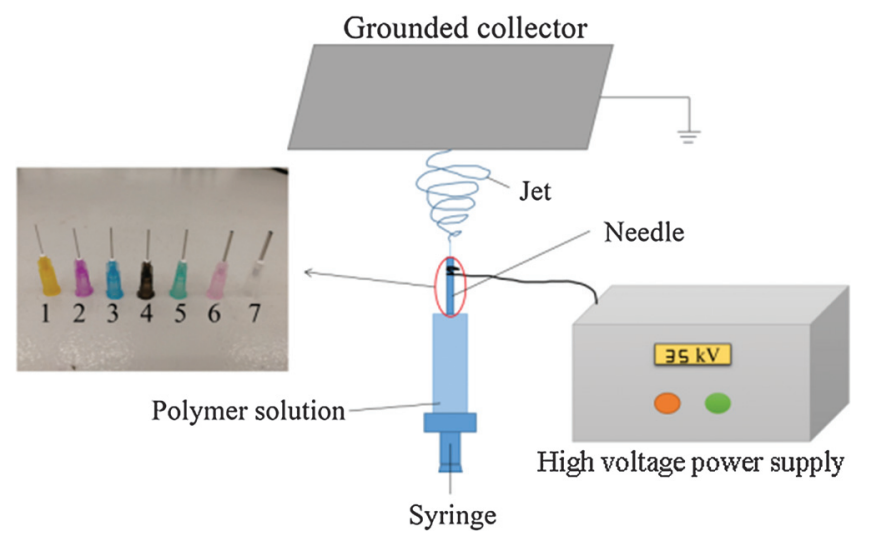

Figure 1. Schematic diagram of single-needle electrospinning setup

Rheological Measurements. The shear viscosity of PEO solutions with concentrations of $3.5 \%, 4.0 \%$, and $4.5 \%$, was evaluated by using a rotational rheometer (AR2000, TA, USA). All samples were analyzed by employing the parallel plate geometry with a diameter of $40 \mathrm{~mm}$, which included a Peltier plate with a planar circular geometry. Flow sweeps were carried out in a shear rate ranging from 1 to $4500 \mathrm{~s}^{-1}$. The gap between the two plates was $600 \mu \mathrm{m}$, and the temperature of the samples during the measurement was set at $15{ }^{\circ} \mathrm{C}, 20{ }^{\circ} \mathrm{C}, 22.5{ }^{\circ} \mathrm{C}, 25{ }^{\circ} \mathrm{C}, 27.5{ }^{\circ} \mathrm{C}, 30{ }^{\circ} \mathrm{C}$, and $35{ }^{\circ} \mathrm{C}$ to investigate how it influences the solution viscosity. Finally, $22.5^{\circ} \mathrm{C}$ was selected as the optimal temperature for rheological property analysis in electrospinning process, because it is the approximate temperature of the electrospinning lab.

Electrospinning. In this study, 7 single needles with the same length of $13 \mathrm{~mm}$ but different inner diameters $(0.51 \mathrm{~mm}$, $0.55 \mathrm{~mm}, 0.63 \mathrm{~mm}, 0.73 \mathrm{~mm}, 0.82 \mathrm{~mm}, 1.2 \mathrm{~mm}$, and $1.32 \mathrm{~mm}$ ) shown in Figure 1 were applied. MA2000 NT 65/P (Hungary)type high voltage power supply was used for the experiments. The voltage and the distance between the grounded metal collector and the needle tip were $35 \mathrm{kV}$ and $20 \mathrm{~cm}$, respectively. The applied solution concentration for needle electrospinning was determined based on the results of rheological measurements and the empirical results from the literature. Solutions were fed using a Aitecs SEP-10S plus (Lithuania) syringe pump with a constant flow (volume) rate and a constant flow velocity (that is related to different flow rates) within the needle.

Scanning Electron Microscopy (SEM). Morphology investigations of nanofibers were carried out by scanning electron microscopy (SEM; JEOL 6380 LA, Japan). Each sample was coated with gold-palladium $(\mathrm{Au}-\mathrm{Pd})$ alloy for $30 \mathrm{~s}$ before the examination. For each sample, 300 fibers were measured to obtain the diameter distribution by using Image $\mathrm{J}$ software.

\section{Results and Discussion}

The Effect of Needle Diameter on Rheological Properties. When a polymer fluid is forced through a needle, the process is shear-rate dominated. Assuming a laminar flow with a constant

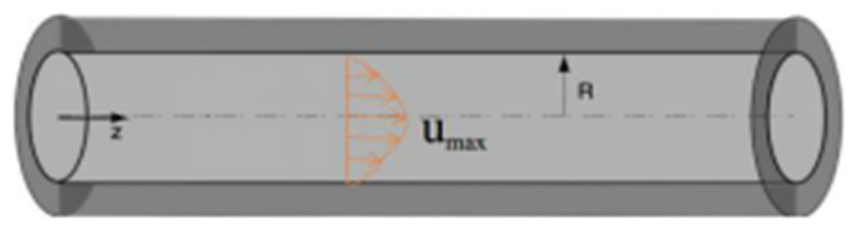

Figure 2. Schematic diagram of laminar flow through a needle flow rate and non-slip boundary conditions, the velocity is maximum $\left(u_{\max }\right)$ at the centerline of the needle and zero at the wall (see Figure 2), making it a non-homogeneous flow.

In the above case, shear rate, $\dot{\gamma}$, is defined as [22] (1):

$$
\dot{\gamma}=\frac{2 u_{\max }}{R}=\frac{4 \bar{u}}{R}
$$

According to the relationship between the velocity and the volumetric flow rate (2):

$$
Q=\pi R^{2} \bar{u}
$$

Finally, the shear rate could be given by (3):

$$
\dot{\gamma}=\frac{4 \bar{u}}{R}=\frac{4 Q}{\pi R^{3}}
$$

where $\dot{\gamma}$ is shear rate, $u_{\max }$ is maximum of flow velocity, $\bar{u}$ is the average flow velocity, $R$ is the needle inner diameter, and $Q$ is the flow rate.

As mentioned above about the flow properties of a solution flowing through a needle, needle diameter, volumetric flow rate, or flow velocity are crucial parameters, which influence shear rate. The shear rates for different needles at the selected flow rates (velocity) are computed and given in Table 1. These needles and flow rates were used in the further experiments.

Table 1. The shear rates introduced by needles used in this study at constant flow rate (A) and constant flow velocity (B) with $4.5 \%$ PEO

\begin{tabular}{llcccc}
\hline $\begin{array}{c}\text { No. Needle diameter } \\
{[\mathrm{mm}]}\end{array}$ & $\begin{array}{c}\text { Needle } \\
\text { color }\end{array}$ & $\begin{array}{c}\text { Flow rate } \\
{[\mathrm{mL} / \mathrm{h}]}\end{array}$ & $\begin{array}{c}\text { Flow velocity } \\
{[\mathrm{mm} / \mathrm{s}]}\end{array}$ & $\begin{array}{c}\text { Shear rate } \\
{\left[\mathrm{s}^{-1}\right]}\end{array}$ \\
\hline \multicolumn{5}{c}{ A: constant flow rate } \\
1 & 0.51 & Orange & $\mathbf{1 . 0}$ & 1.36 & 21.33 \\
2 & 0.55 & Purple & $\mathbf{1 . 0}$ & 1.17 & 17.01 \\
3 & 0.63 & Blue & $\mathbf{1 . 0}$ & 0.89 & 11.32 \\
4 & 0.73 & Black & $\mathbf{1 . 0}$ & 0.66 & 7.27 \\
5 & 0.82 & Green & $\mathbf{1 . 0}$ & 0.53 & 5.13 \\
6 & 1.2 & Pink & $\mathbf{1 . 0}$ & 0.25 & 1.64 \\
7 & 1.32 & Transparent & $\mathbf{1 . 0}$ & 0.20 & 1.23 \\
& \multicolumn{5}{c}{ B: constant flow velocity } \\
8 & 0.51 & Orange & 0.5 & $\mathbf{0 . 6 8}$ & 10.67 \\
9 & 0.55 & Purple & 0.6 & $\mathbf{0 . 6 8}$ & 9.89 \\
10 & 0.63 & Blue & 0.8 & $\mathbf{0 . 6 8}$ & 8.64 \\
11 & 0.73 & Black & 1.0 & $\mathbf{0 . 6 8}$ & 7.45 \\
12 & 0.82 & Green & 1.3 & $\mathbf{0 . 6 8}$ & 6.63 \\
13 & 1.2 & Pink & 2.8 & $\mathbf{0 . 6 8}$ & 4.53 \\
14 & 1.32 & Transparent & 3.4 & $\mathbf{0 . 6 8}$ & 4.12 \\
\hline
\end{tabular}




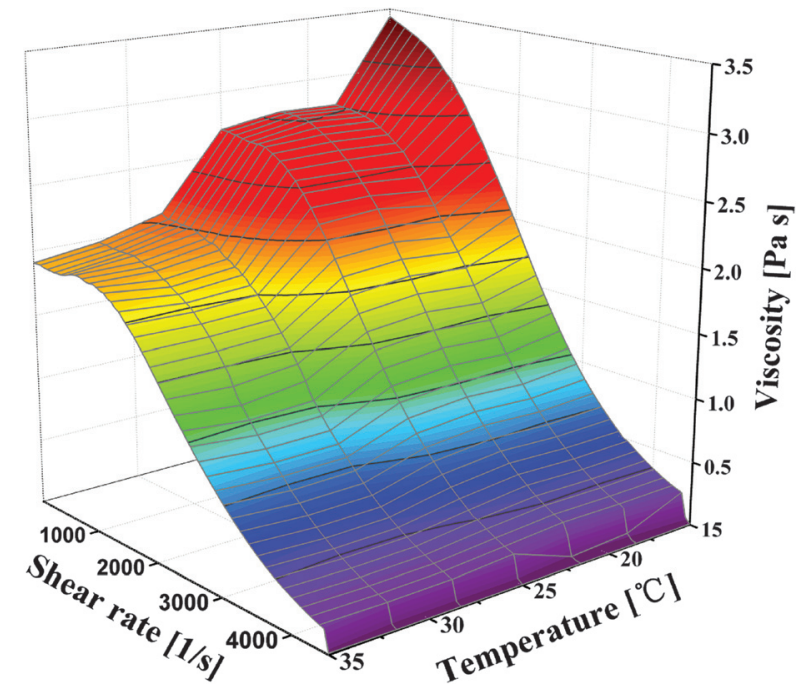

Figure 3. Viscosity of $4.5 \% \mathrm{PEO}$ at different shear rates and temperatures.

Rheological Properties of PEO Solutions. Viscosity is the most widely used material parameter influencing the morphology of nanofibers of electrospinning process. Majority of polymer solutions are shear thinning, and their flow properties are temperature dependent [21]. This means the decrease in viscosity at high rates of deformation and at high temperature. Figure 3 shows the viscosity of $4.5 \%$ PEO solution used in this study. It was obvious that the viscosity of the solution varied greatly under different shear rates and temperatures when the concentration was constant. The reason of higher shear rate resulting in lower viscosity is that at high shear rate, the molecular chains are stretched out, rendering them to slide past one another more easily, which leads to a lower viscosity of the polymer solution. Therefore, it is also the main point of this study that shear rate can also be controlled to adjust solution the viscosity in electrospinning process.

For a non-Newtonian polymer melt, viscosity could be explained by a function of the strain rate and temperature in equation (4) [21],

$$
\eta=m(T) \dot{\gamma}^{n-1}
$$

where $m$ is the consistency index and $n$ is the power law index.

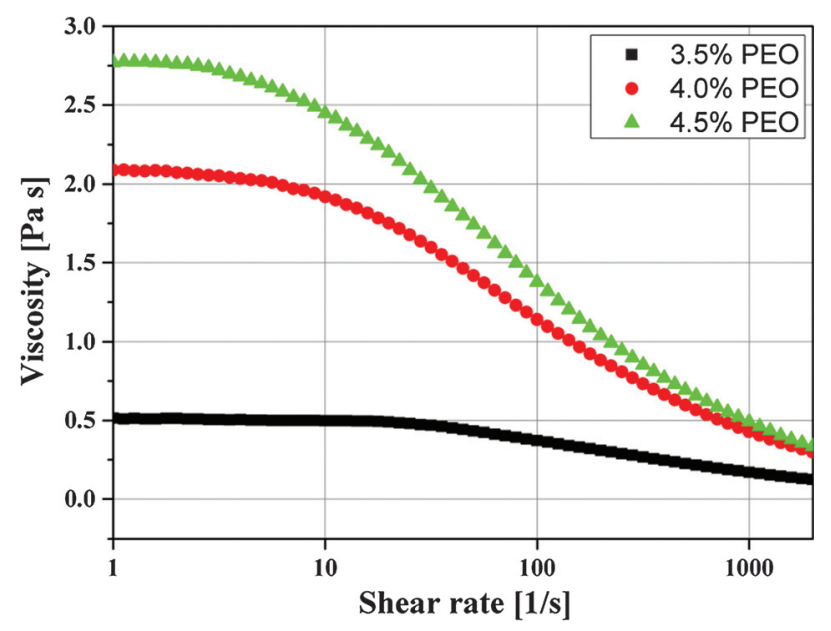

Figure 4. Viscosity curves for PEO solution with different concentrations

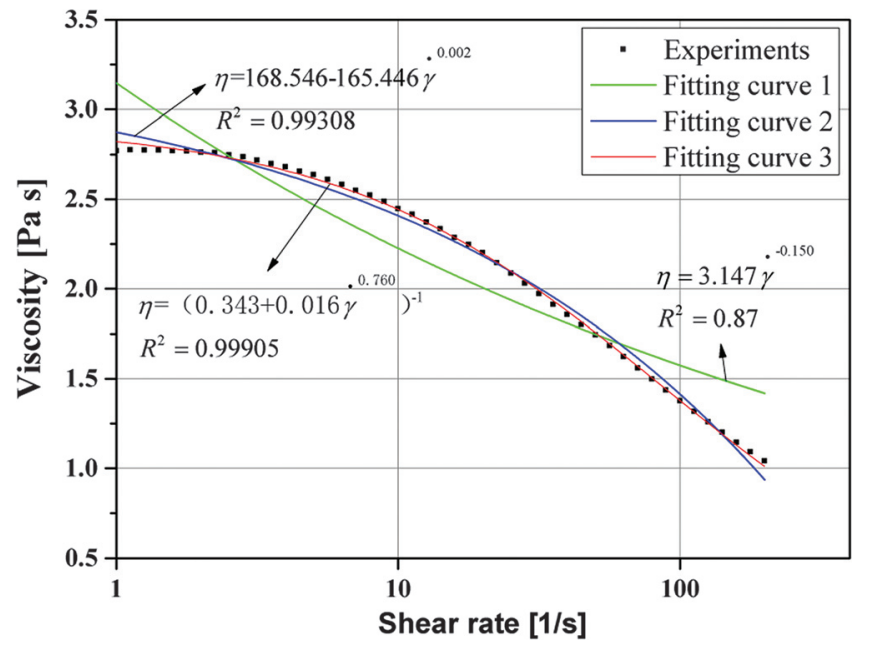

Figure 5. Fitting and experimental viscosity curves of $4.5 \%$ PEO solution

In our experiments, PEO solutions with 3 different kinds of concentration were measured. Figure 4 shows the viscosity curves of PEO solution with different concentrations at $22.5^{\circ} \mathrm{C}$. Based on the result, $4.5 \%$ PEO was used for electrospinning experiments because of the following reasons: (1) it was reported that fiber formation generally occurs when the value of $\eta \cdot C$ is between 5 to 12 [18], in which 3.5\% PEO does not satisfy; and (2) at a same shear rate range, solution with higher concentration has a wider viscosity range, which is good for obtaining obvious results.

The curves of Figure 4 imply that the correlation between shear rate and viscosity of the polymer solution is a kind of a power law function. To carry out the exact formula describing correlation of shear rate and viscosity, 3 different power law methods were used to fit solution viscosity curve. We also tried to fit the curve using equation (4); however, the curve showed a big difference from the experimental data. It revealed that there should be different methods to explain the correlation between shear rate and viscosity for polymer melt and solution. In Figure 5, we can observe that the curve of equation (5) is in good agreement with the experiments; the results obtained from fitting curve can approximate the data from the experiments with the best $R^{2}$ value of 0.99905 (goodness of fit) where $a, b$, and $c$ is $0.343,0.016$, and 0.760 , respectively. Therefore, in a way, the viscosity of the solution flowing through different capillaries can be computed using equation (5), and the viscosity results can be observed in Table 2 .

Table 2. Different parameters (e.g. shear stress, viscosity, fiber diameter) induced by different needles diameters at constant flow rate and flow velocity respectively

\begin{tabular}{lcccc}
\hline No. & $\begin{array}{c}\text { Needle diameter } \\
{[\mathrm{mm}]}\end{array}$ & $\begin{array}{c}\text { Shear rate } \\
{\left[\mathrm{s}^{-1}\right]}\end{array}$ & $\begin{array}{c}\text { Viscosity } \\
{[\mathrm{Pa} \cdot \mathrm{s}]}\end{array}$ & $\begin{array}{c}\text { Fiber diameter } \\
{[\mathrm{nm}]}\end{array}$ \\
\hline \multicolumn{5}{c}{ A: at the constant flow rate } \\
1 & 0.51 & 21.330 & 2.16 & $170.5 \pm 28.0$ \\
2 & 0.55 & 17.006 & 2.26 & $188.3 \pm 20.7$ \\
3 & 0.63 & 11.316 & 2.41 & $179.9 \pm 23.1$ \\
4 & 0.73 & 7.273 & 2.54 & $219.8 \pm 49.5$ \\
5 & 0.82 & 5.132 & 2.64 & $203.9 \pm 25.6$ \\
6 & 1.20 & 1.637 & 2.77 & $218.8 \pm 55.0$ \\
7 & 1.32 & 1.230 & 2.77 & $228.3 \pm 47.6$ \\
& \multicolumn{5}{c}{ B: at the constant flow velocity } \\
8 & 0.51 & 10.667 & 2.44 & $145.8 \pm 22.8$ \\
9 & 0.55 & 9.891 & 2.46 & $151.5 \pm 42.8$ \\
10 & 0.63 & 8.635 & 2.50 & $165.7 \pm 23.3$ \\
11 & 0.73 & 7.452 & 2.54 & $177.8 \pm 36.3$ \\
12 & 0.82 & 6.634 & 2.58 & $191.1 \pm 50.7$ \\
13 & 1.2 & 4.533 & 2.66 & $202.3 \pm 50.0$ \\
14 & 1.32 & 4.121 & 2.68 & $194.2 \pm 54.7$ \\
\hline
\end{tabular}



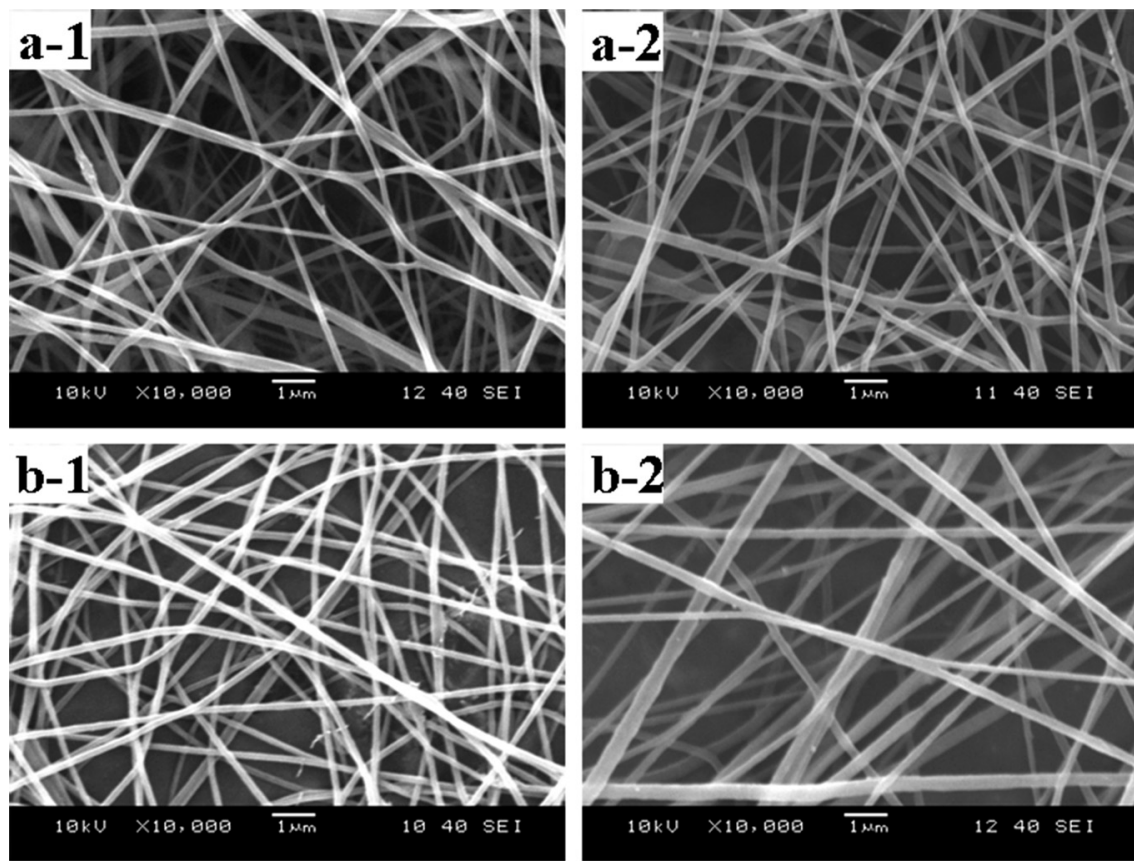

Figure 6. SEM images of 4.5\% PEO nanofiber samples with different needle diameters of $0.51 \mathrm{~mm}(1)$ and $1.32 \mathrm{~mm}(2)$ at a constant flow rate (a) and a constant flow velocity (b), respectively

$$
\eta=\left(a+b \dot{\gamma}^{c}\right)^{-1}
$$

The Effect of Needle Diameter on Fiber Morphology and Diameter. SEM micrographs of PEO nanofibers obtained from needles with different inner diameters at a constant flow rate and a constant flow velocity are presented in Figure 6. It is observed that thinner fibers were obtained using a smaller diameter needle (all other parameters were kept constant).

Furthermore, the diameters of the resulting fibers were analyzed statistically by calculating the average diameter and their standard deviation in order to investigate the correlation between the fiber diameter and needle diameter. The results shown in Figure $7 \mathrm{~b}$ indicate that an increase in the needle diameter resulted in an increase in average fiber diameter for both constant flow rate and velocity. The reason behind the phenomenon is that the solution viscosity is affected by needle diameter. Based on the rheological analysis of polymer solution flowing in a needle, the relationship between the needle diameter and the solution viscosity can be obtained and depicted in Figure 7a. These results are in line with the accepted fact that higher viscosity can result in thicker fiber. As the needle diameter was $0.51 \mathrm{~mm}$, it could provide a shear rate of $21.330 \mathrm{~s}^{-1}$ (at a constant flow rate) and $10.667 \mathrm{~s}^{-1}$ (at a constant flow velocity) which could make the solution viscosity decrease from 2.77 $\mathrm{Pa} \cdot \mathrm{s}$ (zero-shear viscosity) to $2.16 \mathrm{~Pa} \cdot \mathrm{s}$ and $2.44 \mathrm{~Pa} \cdot \mathrm{s}$, respectively (Table 2 ). Lower viscosity can render the polymer easier to be stretched into a fiber due to the less resistance of molecular chains. Additionally, the results exhibited that the fiber diameter distribution becomes wider by increasing the needle diameter (Figure 8). Therefore, the needle with smaller diameter is suggested for the capability of fabricating thinner nanofibers. The presented results are in contrast to those reported in the literature, where it was concluded that there was no correlation between the needle diameter and fiber diameter [10]. Because in the literature, only three different needles were investigated, moreover the viscosity difference caused by needle diameter were also neglected.

\section{Conclusions}

To summarize, the reason of the effect of needle diameter on fiber diameter was analyzed in detail from the view of rheology. The results indicated that fiber diameter and
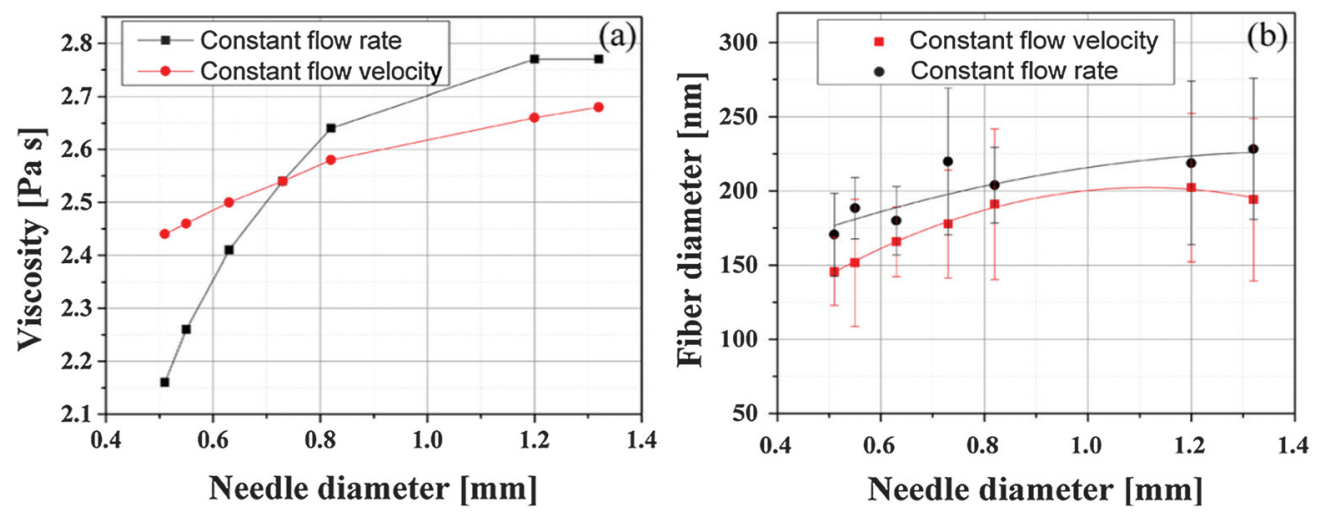

Figure 7. Viscosity of 4.5\% PEO (a) and diameter of nanofibers (b) with different needle diameters at a constant flow rate and a constant flow velocity 

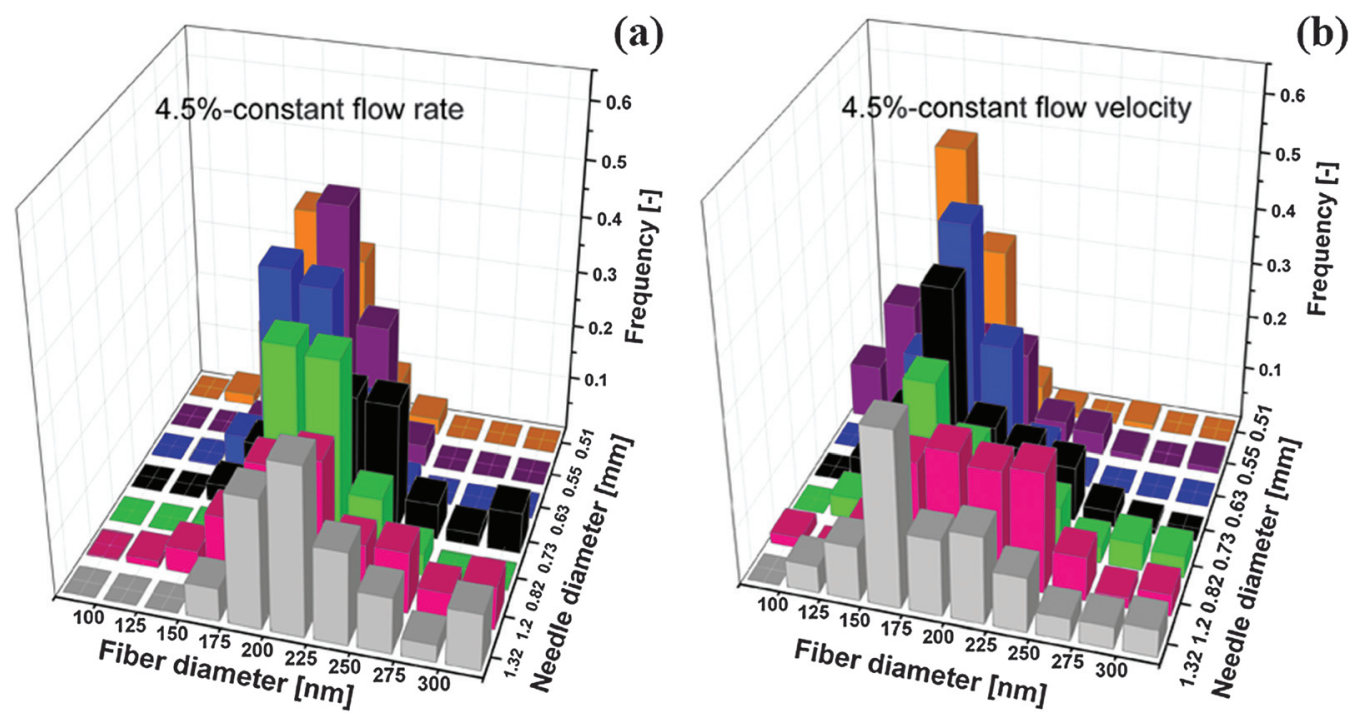

(b)

Figure 8. Fiber diameter distribution at a constant flow rate (a) and a constant flow velocity (b)

morphology strongly depend on needle diameter because of the different shear rates originating from needle diameter. It is obvious that the viscosity of polymer materials is an important parameter in polymer processing, which not only is controlled by some inherent (static) factors (e.g., molecular mass and concentration), but also can be changed by dynamic factors (e.g., shear rate and temperature). However, in the field of electrospinning, most research studies just focused on inherent (static) factors, which is not suitable to investigate continuously changing parameters. Hopefully, the method of changing the viscosity of polymer materials by dynamic factors can provide a new thought and a valuable reference for electrospinning.

Acknowledgments. This research was supported by the Hungarian Research Fund (OTKA K100949, PD116122) and by the ÚNKP-17-4-I New National Excellence Program of the Ministry of Human Capacities and BME-KKP and China Scholarship Council (201700500073).

Open Access. This is an open-access article distributed under the terms of the Creative Commons Attribution-NonCommercial 4.0 International License (https://creativecommons.org/licenses/ by-nc/4.0/), which permits unrestricted use, distribution, and reproduction in any medium for non-commercial purposes, provided the original author and source are credited, a link to the CC License is provided, and changes - if any - are indicated.

\section{References}

1. MacDiarmid, A. G.; Jones, W. E.; Norns, I. D.; Gao, J.; Johnson, A. T; Pinto, N. J.; Hone, J.; Han, B.; Ko, F. K.; Okuzaki, H.; Llaguno, M. Synth. Met. 2001, 119, 27-30

2. Lee, S. H.; Ku, B. C.; Wang, W. Mat. Res. Soc. Symp. Pro. 2002, 708, 403-408.

3. Buchko, C. J.; Chen, L. C.; Shen, Y.; Martin, D. C. Polymer 1999, 40, 7397-7407.

4. Kenawy, E.; Bowlin, G. L.; Mansfield, K.; Simpson, D. G.; Wnek, G. E. J. Contr. Release 2002, 81, 57-64.

5. Márquez, Y.; Graupera, J.; del Valle, L. J.; Turon, P.; Franco, L.; Puiggalí, J. eXPRESS Polym. Lett. 2017, 11, 674-689.

6. Lomov, S. V.; Molnár, K. eXPRESS Polym. Lett. 2016, 10, 25-35.

7. Mokhena, T. C.; Jacobs, V.; Luyt, A. S. eXPRESS Polym. Lett. 2017, $11,652-663$.

8. Stepanyan, R.; Subbotin, A. V.; Cuperus, L.; Boonen, P.; Dorschu, M.; Oosterlinck, F.; Bulters, M. J. H. Polymer 2016, 97, 428-439.

9. Subbiah, T.; Bhat, G. S.; Tock, R. W. J. Appl. Polym. Sci. 2005, 96, 557-569.

10. Macossay, J.; Marruffo, A.; Rincon, R.; Eubanks, T.; Kuang, A. Polym. Adv. Technol. 2007, 18, 180-183.

11. Fong, H.; Chun, I; Reneker, D. H. Polymer 1999, 40, 4585-4592.

12. Deitzel, J. M.; Kleinmeyer, J.; Harris, D.; Beck Tan, N. C. Polymer 2001, 42, 261-272.

13. Heikkilä, P.; Harlin, A. Eur. Polym. J. 2008, 44, 3067-3079.

14. Bhardwaj, N.; Kundu, S. C. Biotechnol. Adv. 2010, 28, 325-347.

15. Bo Kyung, P.; In Chul, U. Int. J. Biol. Macromol. 2018, 106, 1166-1172.

16. Tacx, J. C. J. F.; Schoffeleers, H. M.; Brands, A. G. M.; Teuwen, L. Polymer 2000, 41, 947-957.

17. Tao, J.; Shivkumar, S. Mater. Lett. 2007, 61, 2325-2328.

18. Tsou, S. Y.; Lin, H. S.; Wang, C. Polymer 2011, 52, 3127-3136.

19. Theron, S. A.; Zussman, E.; Yarin, A. L. Polymer 2004, 45 2017-2030.

20. Son, W. K.; Youk, J. H.; Lee, T. S.; Park, W. H. Polymer 2004, 45, 2959-2966.

21. Osswald, T. A. Understanding Polymer Processing, Germany, Hanser Publisher, 2010, pp. 57.

22. Darby, R. Chemical Engineering Fluid Mechanics (2nd ed.), CRC Press, 2001, pp. 64. 